Int. J. Dev. Biol. 61: 557-563 (2017)

doi: $10.1387 / \mathrm{ijdb} .170155 \mathrm{jb}$

\title{
Temporal and spatial expression patterns of Hedgehog receptors in the developing inner and middle ear
}

\author{
JEONG-OH SHIN ${ }^{1, \#, ~ H A R I N A R A Y A N A ~ A N K A M R E D D Y ~}{ }^{1,3, \#,}$ NAGA MAHESH JAKKA ${ }^{1}$, SEOKWON LEE ${ }^{4,5}$, \\ UN-KYUNG KIM ${ }^{4,5}$ and JINWOONG BOK*,1,2,3

\begin{abstract}
${ }^{1}$ Department of Anatomy, ${ }^{2}$ Department of Otorhinolaryngology, ${ }^{3}$ BK21 PLUS Project for Medical Science, Yonsei University College of Medicine, Seoul, ${ }^{4}$ Department of Biology, College of Natural Sciences and ${ }^{5}$ School of Life Sciences, BK21 Plus KNU Creative BioResearch Group, Kyungpook National University, Daegu, Republic of Korea
\end{abstract}

\begin{abstract}
The mammalian inner ear is a complex organ responsible for balance and hearing. Sonic hedgehog (Shh), a member of the Hedgehog (Hh) family of secreted proteins, has been shown to play important roles in several aspects of inner ear development, including dorsoventral axial specification, cochlear elongation, tonotopic patterning, and hair cell differentiation. Hh proteins initiate a downstream signaling cascade by binding to the Patched 1 (Ptch1) receptor. Recent studies have revealed that other types of co-receptors can also mediate $\mathrm{Hh}$ signaling, including growth arrest-specific 1 (Gas1), cell-adhesion molecules-related/down-regulated by oncogenes (Cdon), and biregional Cdon binding protein (Boc). However, little is known about the role of these Hh co-receptors in inner ear development. In this study, we examined the expression patterns of Gas1, Cdon, and Boc, as well as that of Ptch1, in the developing mouse inner ear from otocyst (embryonic day (E) 9.5) until birth and in the developing middle ear at E15.5. Ptch 1, a readout of Hh signaling, was expressed in a graded pattern in response to Shh signaling throughout development. Expression patterns of Gas1, Cdon, and Boc differed from that of Ptch1, and each Hh co-receptor was expressed in specific cells and domains in the developing inner and middle ear. These unique and differential expression patterns of $\mathrm{Hh}$ co-receptors suggest their roles in mediating various time- and space-specific functions of Shh during ear development.
\end{abstract}

KEY WORDS: inner ear, hedgehog, Gas1, Cdon, Boc

The mammalian hearing apparatus consists of the outer, middle, and inner ear. The inner ear is derived from thickened surface ectoderm (also known as the otic placode) on either side of the hindbrain. The otic placode invaginates to form the otocyst, which undergoes a series of morphogenetic changes to produce the threedimensional structure of the inner ear. The inner ear is composed of six sensory organs, including three cristae (anterior, lateral, and posterior) and two maculae (utricle and saccule) in the vestibule and the organ of Corti in the cochlea. Proper ear development requires multiple signaling molecules, some of which emanate from external sources, such as the neural tube, notochord, and periotic mesenchyme, whereas others are expressed within the otic epithelium itself (Wu and Kelley, 2012).

Sonic hedgehog (Shh) is one such signaling molecule that emanates from surrounding tissues to contribute to multiple steps of inner ear development. During the otocyst stage, Shh is expressed from the floor plate and notochord and specifies the dorsoventral axis of the otocyst, which regionalizes the inner ear into dorsal vestibular and ventral cochlear compartments (Bok et al., 2005, Bok et al., 2007, Brown and Epstein, 2011, Riccomagno et al., 2002). In addition, Shh expressed from spiral ganglion neurons (SGNs) regulates the timing of hair cell differentiation and cochlear elongation (Bok et al., 2013, Tateya et al., 2013). A recent study demonstrated that Shh signaling gradient confers regional identities

\footnotetext{
Abbreviations used in this paper: Bmp4, bone morphogenetic protein 4; Boc, biregional cdon binding protein; Cdon, cell-adhesion molecule-related/down-regulated by oncogenes; CVG, cochleovestibular ganglia; Gas 1, growth arrest-specific 1; Hh, hedgehog; Lfng, LFNG O-fucosylpeptide 3-beta-N-acetylglucosaminyltransferase; Shh, sonic hedgehog; Ptch 1, patched 1; SGN, spiral ganglion neuron.
}

\footnotetext{
*Address correspondence to: Jinwoong Bok. Department of Anatomy, Yonsei University College of Medicine, Seoul, 03722, Republic of Korea. Fax: +82-2-365-0700. Tel: +82-2-2228-0753. E-mail: bokj@yuhs.ac iD http://orcid.org/0000-0003-1958-1872
}

\#Note: These authors contributed equally to this work. 

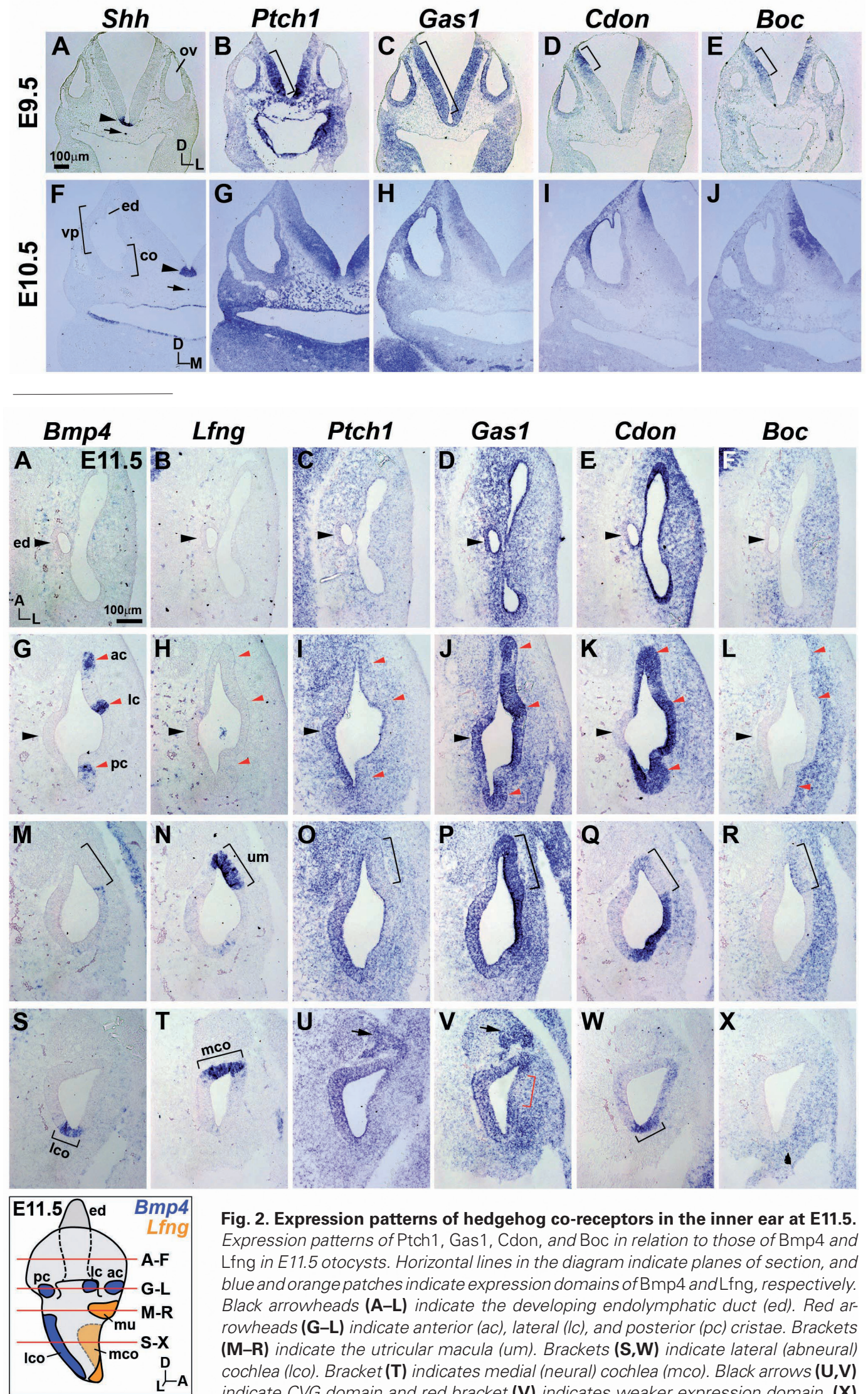

Fig. 2. Expression patterns of hedgehog co-receptors in the inner ear at E11.5. Expression patterns of Ptch1, Gas1, Cdon, and Boc in relation to those of Bmp4 and Lfng in E11.5 otocysts. Horizontal lines in the diagram indicate planes of section, and blue and orange patches indicate expression domains of Bmp4 and Lfng, respectively. Black arrowheads (A-L) indicate the developing endolymphatic duct (ed). Red arrowheads (G-L) indicate anterior (ac), lateral (Ic), and posterior (pc) cristae. Brackets (M-R) indicate the utricular macula (um). Brackets (S, W) indicate lateral (abneural) cochlea (Ico). Bracket (T) indicates medial (neural) cochlea (mco). Black arrows (U,V) indicate CVG domain and red bracket (V) indicates weaker expression domain. (X) Boc is not expressed in the ventral otic epithelium. A, anterior; L, lateral; D, dorsal.
Fig. 1. Expression patterns of hedgehog co-receptors in the otocyst at E9.5 and E10.5. Expression patterns of Shh, Ptch1, Gas1, Cdon, and Boc transcripts in the neural tube and otic epithelium at E9.5 (A-E) and E10.5 (F-J). Square brackets (B-E) indicate expression domains of $\mathrm{Hh}$ co-receptors in the neural tube. Arrowheads and arrows $(\mathbf{A}, \mathbf{F})$ indicate Shh expression in the floor plate and notochord, respectively. ov, otic vesicle; co, cochlear duct; ed, endolymphatic duct; vp, vertical pouch; $D$, dorsal; L, lateral; $M$, medial.

to the developing cochlea, which prefigures the tonotopic organization of the mature cochlea (Son et al., 2015).

It is interesting that a single signaling molecule such as Shh exerts such a broad range of functions in a precisely controlled time- and space-specific manner to build a functional inner ear. It is generally accepted that binding of $\mathrm{Hh}$ ligands to the receptor Ptch1 activates the key signaling mediator Smo, which initiates a downstream signaling cascade leading to transcriptional regulation by Gli transcription factors (Briscoe and Therond, 2013). Recently, other Hhco-receptors have been shown to play essential roles in mediating Hh signaling. These co-receptors include growth arrest-specific 1 (Gas1), cell-adhesion molecule (CAM)-related/down-regulated by oncogenes (Cdon), and biregional Cdon binding protein (Boc) (Allen et al., 2011). Whereas Gas1 is a vertebrate-specific gene, Cdon and $B O C$ are conserved from Drosophila to mouse. Cdon and Boc are homologs of Drosophila interference hedgehog (Ihog) and brother of Ihog (Boi), respectively, which are functionally and structurally redundant, and are essential for Hh signaling in Drosophila (Camp et al., 2010). In mice, Hh co-receptors interact with Ptch1, forming distinct receptor complexes to regulate $\mathrm{Hh}$-mediated function, and simultaneous deletion of Gas1, Cdon, and Boc results 
in phenotypes resembling those of Shh null mutants (Allen et al., 2011, Izzi et al., 2011, Tenzen et al., 2006). These findings have raised the possibility that $\mathrm{Hh}$ co-receptors may mediate various Shh functions required for inner ear development. In this study, we analyzed temporal and spatial expression patterns of Gas1, Cdon, and Boc in the developing inner and middle ear. We observed that each Hh co-receptor was expressed in specific cells or domains, suggesting that distinct combinations of Hh receptor complexes mediate various Shh functions in a time- and space-dependent manner during ear development.

\section{Results and Discussion}

\section{Expression patterns of hedgehog co-receptors during early otocyst stages}

Expression patterns of Gas1, Cdon, and Bocin the otocyst were examined together with those of Shh and Ptch1 at E9.5 and E10.5 (Fig. 1). Shh was expressed in ventral midline structures, such as the floorplate and notochord (Fig. 1 A,F). Ptch1 was expressed in a graded pattern in the otocyst and neural tube, more strongly on the ventral side and becoming gradually weaker toward the dorsal side (Fig. $1 \mathrm{~B}, \mathrm{G})$, representing a Shh signaling gradient emanating from the ventral midline (Bok et al., 2007). Unlike Ptch1, Gas1 was broadly expressed in the otocyst and the neural tube at E9.5, and then was restricted to the dorsal half of the otocyst and neural tube at E10.5 (Fig. $1 \mathrm{C}, \mathrm{H}$ ). Cdon was expressed on the dorsolateral side of the otocyst and in the dorsal tip of the neural tube at E9.5 and E10.5 (Fig. 1 D,I). Expression patterns of Gas1 and Cdon at E10.5 were generally complementary to that of Ptch1 (Fig. 1 $\mathrm{G}-\mathrm{I})$, consistent with the idea that Gas1 and Cdon are negatively regulated by Shh signaling (Allen et al., 2011, Martinelli and Fan, 2007, Tenzen et al., 2006). In contrast, Boc expression was not observed in the otocyst at E9.5 and E10.5 (Fig. 1 E,J), and was localized in the middle region between the areas of Ptch1 and Cdon expression in the neural tube (Fig. 1). Gas1, Cdon, and Boc have been shown to play overlapping and essential roles in Shh-mediated ventral patterning of the neural tube by forming distinct receptor complexes with Ptch1 (Allen et al., 2011, Izzi et
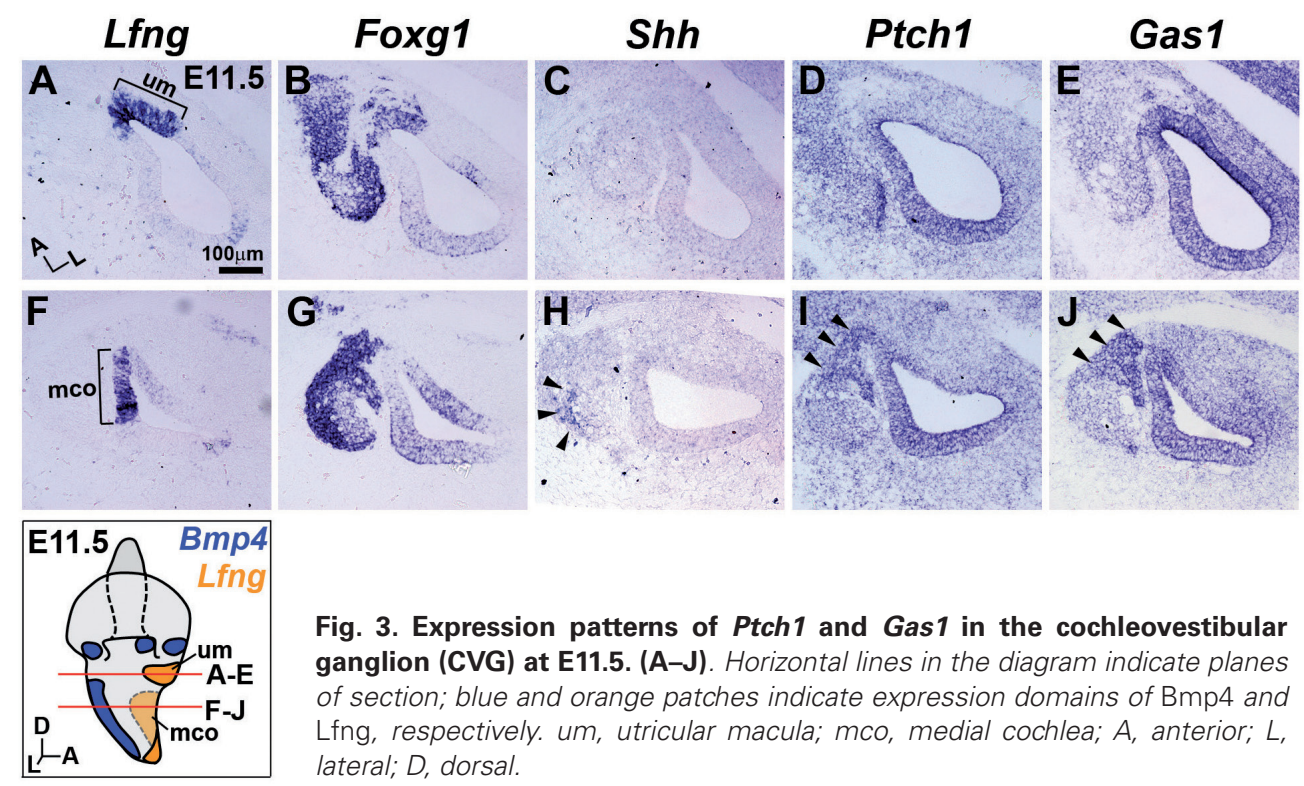

2011). Comparable patterns of expression of Hh co-receptors in the developing otocyst and neural tube suggest essential roles of $\mathrm{Hh}$ co-receptors in mediating Shh functions that are required during early inner ear development, such as dorsoventral axial specification (Bok et al., 2007).

\section{Expression patterns of hedgehog co-receptors in the presumptive sensory regions in the E11.5 otocyst}

We next examined expression patterns of $\mathrm{Hh}$ co-receptors at E11.5 in relation to the presumptive sensory organs, which can be identified by expression of the markers such as bone morphogenetic protein 4 (Bmp4) in cristae and lateral (abneural) cochlea and Lunatic Fringe ( $L f n g$ ) in utricular macula and medial (neural) cochlea (Fig. 2) (Morsli et al., 1998). Ptch1 was expressed in a graded pattern, more weakly in the dorsal vestibular organs and becoming gradually stronger toward the ventral cochlea (Fig. 2 $\mathrm{C}, \mathrm{I}, \mathrm{O}, \mathrm{U})$. In contrast, Gas1 was broadly expressed in the otic epithelium and periotic mesenchyme without a clear gradient (Fig. 2 $\mathrm{D}, \mathrm{J}, \mathrm{P}, \mathrm{V})$, suggesting that, unlike Ptch1, Gas 1 is expressed independently of Shh signaling at this stage. Interestingly, Gas1 expression was absent in a small region in the lateral cochlea (Fig. 2V, red bracket), which appears to later develop into the stria vascularis, based on Gas1 expression at E15.5. Gas1 was also expressed in the developing cochleovestibular ganglion (CVG) in a pattern similar to that of Ptch1 (Fig. $2 \mathrm{U}, \mathrm{V}$, arrows). Cdon was expressed in a gradient opposite that of Ptch1 (stronger dorsally and weaker ventrally) in the otic epithelium and periotic mesenchyme (Fig. 2 $\mathrm{E}, \mathrm{K}, \mathrm{Q}, \mathrm{W})$, suggesting negative regulation of $C$ don by Shh signaling. Boc expression was not observed in the otic epithelium, with the exception of an area of weak expression in the utricular macula (Fig. 2 F,L,R,X, bracket). Our results showed that each Hh receptor exhibits unique expression patterns in the E11.5 otocyst. For example, Gas1 and Cdon were expressed in the cristae, whereas Gas1 and Boc were expressed in the utricular macula. In addition, expression domains of Cdon and Boc demarcated a sharp boundary between the presumptive utricular macula and non-sensory epithelium (Fig. $2 \mathrm{Q}, \mathrm{R}$, brackets). It will be interesting to examine whether distinct combinations of $\mathrm{Hh}$ co-receptors contribute to fate determination of specific sensory organs, such as cristae or maculae.

Expression patterns of Ptch1 and Gas1 in the cochleovestibular ganglion

During early inner ear development, neuroblasts delaminate from the otic epithelium to form the CVG, which later connects hair cells to the brain. We analyzed expression of Hh co-receptors in the CVG, which was identified by Foxg1 expression in the mesenchyme located anteromedial to the otocyst (Fig. $3 \mathrm{~B}, \mathrm{G}$ ). Shh expression was observed in the CVG only at the cochlear level and not at the utricular level (Fig. 3 $\mathrm{C}, \mathrm{H}$, arrowheads) (Boketal., 2013). Consistently, Ptch1 and Gas 1 were upregulated in the CVG located near
Fig. 3. Expression patterns of Ptch1 and Gas1 in the cochleovestibular ganglion (CVG) at E11.5. (A-J). Horizontal lines in the diagram indicate planes of section; blue and orange patches indicate expression domains of Bmp4 and Lfng, respectively. um, utricular macula; mco, medial cochlea; $A$, anterior; L, lateral; $D$, dorsal. 
the Shh-expressing population only at the cochlear level (Fig. 3D, E, I, and J, arrowheads). Neither Cdon nor Boc was expressed in the CVG (Fig. $2 \mathrm{~W}, \mathrm{X}$ ). Although the roles of ganglionic Shh in hair cell differentiation and cochlear elongation have been well documented (Bok et al., 2013), its role in CVG development is less clear. It will be interesting to examine whether Ptch1 and Gas1 mediate Shh functions that are required for CVG development, such as the mitogenic role previously noted in cerebellar granular neuronal progenitors (Izzi et al., 2011).

\section{Expression patterns of Hh co-receptors in the inner ear at E15.5}

Expression patterns of Hh co-receptors were examined at E15.5 when the developing inner ear attains its mature morphology (Fig. $4 \mathrm{~A}$ ) and $L$ fng was expressed in all six sensory organs (Fig. 4 E-F') (Morsli et al., 1998). Shh was expressed in the SGNs in the apical turn (Fig.4 B, arrow), and Ptch1 was expressed in the cochlear epithelium and adjacent mesenchyme in a graded pattern that was stronger in the apex and weaker toward the base (Fig. 4D) (Bok et al., 2013). No detectable expression of Ptch1 was observed in the vestibule (Fig. 4C). Gas1 was broadly expressed in the inner ear, including the sensory organs, but not in the roof of the utricular macula (Fig. 4G) and the developing stria vascularis (Fig. $4 \mathrm{H}-\mathrm{H}^{\prime}$ ), which, interestingly, lacked Gas1 expression at E11.5 (Fig. 2V). In contrast, Cdon was not expressed in the sensory organs, but was expressed in most of the non-sensory regions, with the exception of the endolymphatic duct and Reissner's membrane (Fig. 4J'). Cdon expression in the cochlea was graded in a pattern that was stronger at the base and weaker toward the apex, which was opposite that of Ptch1 (Fig. $4 \mathrm{D}, \mathrm{J}$ ). Boc was weakly expressed in the sensory regions and surrounding mesenchyme in the vestibule (Fig. $4 \mathrm{~K})$ and in the Hansen and Claudius cells and greater epithelial ridge (GER) in the cochlea (Fig. 4L'). Our findings showed that the spatial relationships between sensory regions and the expression domains of $\mathrm{Hh}$ co-receptors are generally maintained from the otocyst at E11.5 to the inner ear at E15.5, suggesting that $\mathrm{Hh}$ coreceptors may be involved in establishment and maintenance of boundaries between sensory and non-sensory regions or between specific inner ear structures, such as Reissner's membrane and stria vascularis.

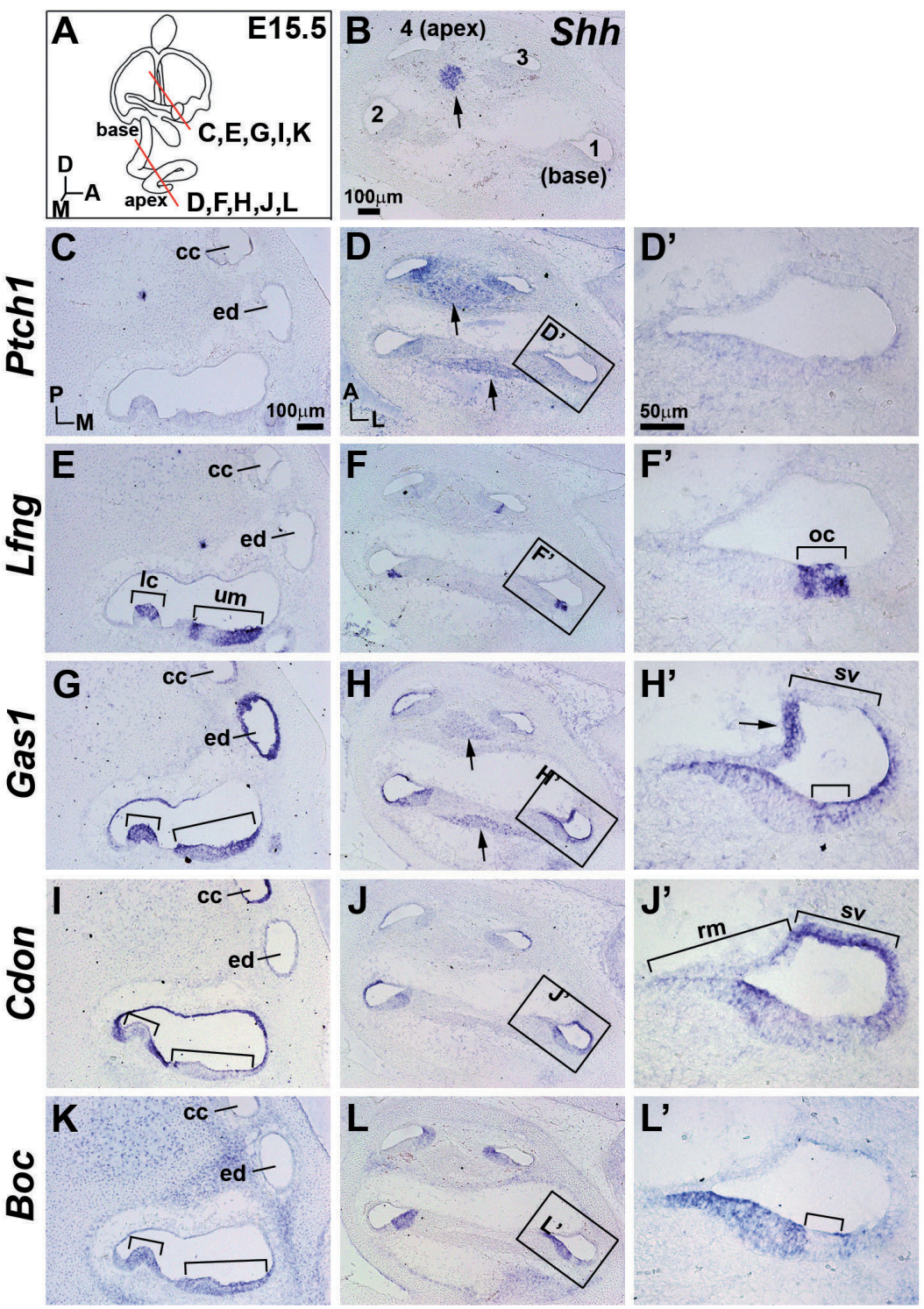

\section{Expression patterns of Hh co-receptors in hair cells in the neonatal inner ear}

We next examined expression patterns of $\mathrm{Hh}$ coreceptors in the neonatal inner ear together with that of the hair cell marker atonal bHLH transcription factor 1 (Atoh1) (Fig. 5 A-B", arrowheads). No Ptch1 expression was observed in the inner ear, indicating that little, if any, Shh signaling takes place in the inner ear at this stage (Fig. 5 C-D'). In contrast, Gas1 was strongly expressed in the vestibular sensory regions, most likely in supporting cells, based on the comparison between Atoh1 and Gas1 expression patterns in the cristae and maculae (Fig. 5 A-A" and E-E"). In the cochlea, Gas1 was broadly expressed in the outer and inner sulcus cells, Reissner's membrane, and supporting cells abutting the hair cells (Fig. 5 F,F'). Unlike E11.5 and E15.5, Gas 1 was expressed in the mesenchyme adjacent to the developing stria vascularis, which appeared to be the differentiating strial basal cell layer (Song et al., 2011). Expression domains of $C$ donat P0 were continuous with those seen at E15.5, such as in non-sensory

Fig. 4. Expression patterns of hedgehog co-receptors in the inner ear at E15.5. (A) Red lines in the diagram indicate planes of section. (B) Shh expression in the spiral ganglion neuron (SGN) of the apical cochlear turn (arrow). Numbers (1-4) indicate the cochlear turns from base to apex. Expression of Ptch1, Lfng, Gas1, Cdon, and Boc in the vestibule (C. $\mathbf{E}, \mathbf{G}, \mathbf{I}, \mathbf{K})$ and the cochlear $\operatorname{duct}(\mathbf{D}, \mathbf{F}, \mathbf{H}, \mathbf{J}, \mathbf{L})$. Arrows (D, H) indicate expression of Ptch1 and Gas1 in the SGN. The basal cochlear turn is enclosed by black lines and is shown at higher magnification in $\mathbf{D}^{\prime}, \mathbf{F}^{\prime}, \mathbf{H}^{\prime}, \mathbf{J} \mathbf{J}^{\prime}, \mathbf{L}^{\prime}$. Small and large brackets (E, G, $\mathbf{I}, \mathbf{K})$ indicate the lateral crista (Ic) and utricular macula (um), respectively. Small brackets $\left(\mathbf{F}^{\prime}, \mathbf{H}^{\prime}, \mathbf{J}^{\prime}, \mathbf{L}^{\prime}\right)$ indicate the organ of Corti (oc). Larger brackets $\left(\mathbf{H}^{\prime}\right.$ and $\left.\mathbf{J}^{\prime}\right)$ represent the stria vascularis (sv) and Reissner's membrane (rm). cc, common crus; ed, endolymphatic duct. A, anterior; $D$, dorsal; $M$, medial; $P$, posterior. Scale bar shown in B applies to panels B-L; Scale bar shown in $D^{\prime}$ applies to panels $D^{\prime}-L^{\prime}$. 


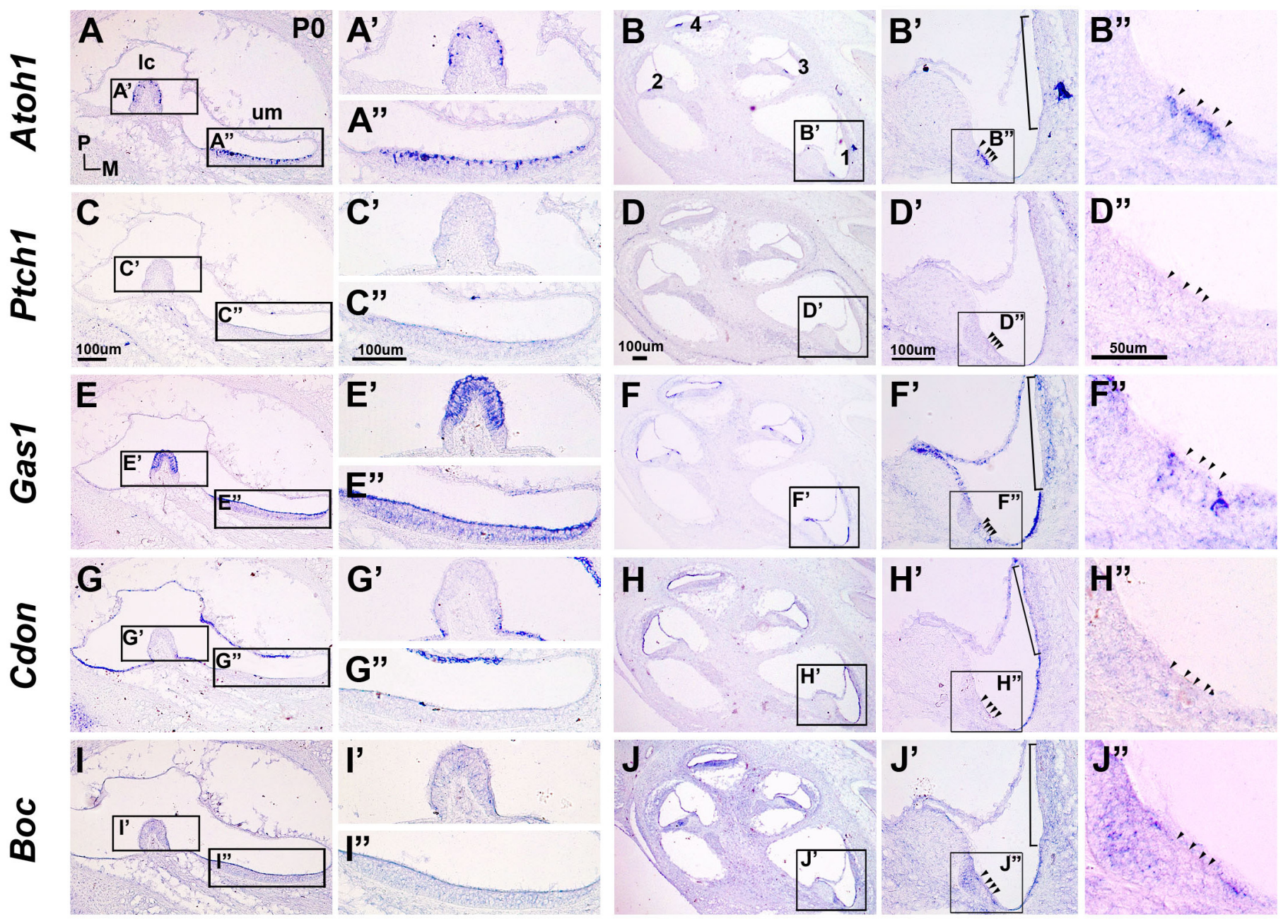

Fig. 5. Expression patterns of hedgehog co-receptors in the inner ear at P0. Expression patterns of Atoh1, Ptch1, Gas1, Cdon, and Boc in vestibule $(\mathbf{A}, \mathbf{C}, \mathbf{E}, \mathbf{G}, \mathbf{I})$ and cochlea (B, D, F, H, J). The lateral crista (IC) and utricular macula (um) are enclosed by black lines and are shown at higher magnification in $\mathbf{A}^{\prime}, \mathbf{C}^{\prime}, \mathbf{E}^{\prime}, \mathbf{G}$ ', I' and $\mathbf{A}^{\prime \prime}, \mathbf{C}^{\prime \prime}, \mathbf{E}^{\prime \prime}, \mathbf{G}$ ", and I", respectively. Numbers (1-4) in B indicate the cochlear turns from base to apex. The basal cochlear turn is enclosed by black lines and is shown at higher magnification in $\mathbf{B}^{\prime}, \mathbf{D}^{\prime}, \mathbf{F}^{\prime}, \mathbf{H}^{\prime}$, and $\mathbf{J}^{\prime}$; in these panels the organ of Corti is enclosed in black lines and is shown at higher magnification in $\mathbf{B}$ ", D", F", H", and J". Arrowheads indicate the hair cells in the organ of Corti. Scale bars apply to all panels in a same vertical column of images.

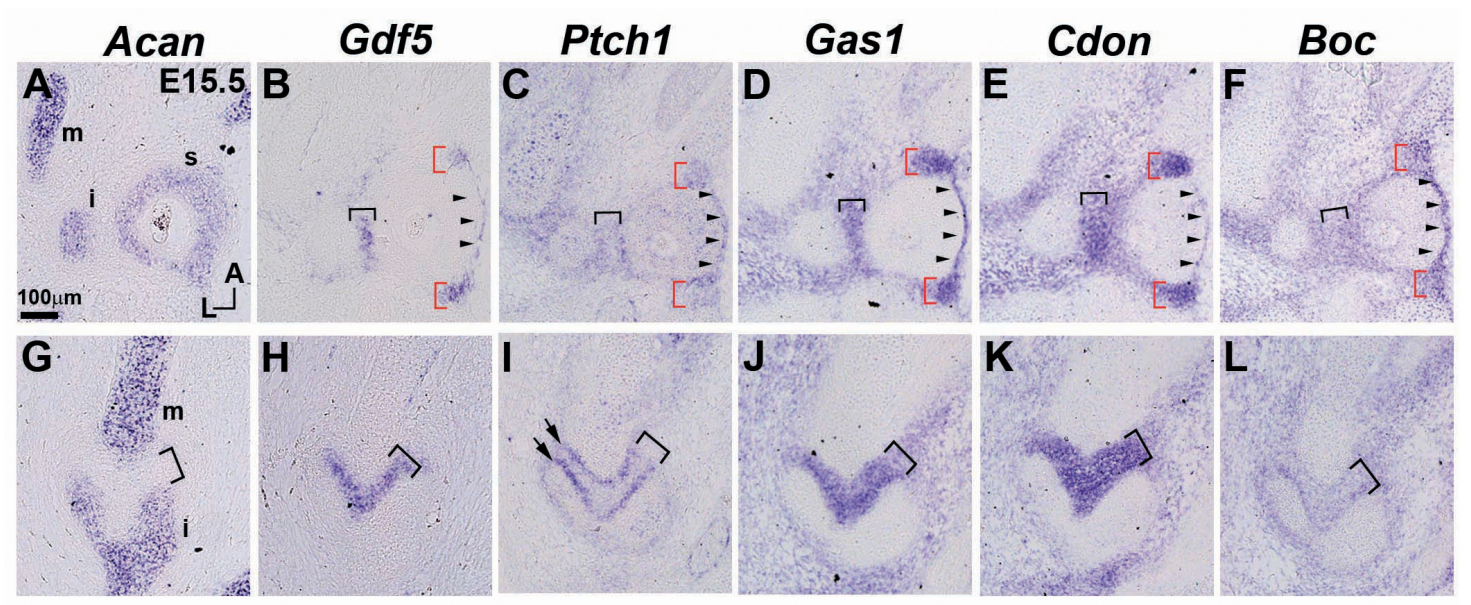

Fig. 6. Expression patterns of hedgehog co-receptors in the middle ear. Acan is expressed in the middle ear ossicles (A,G), and Gdf5 is expressed in the joints and annular ligaments (B,H). Expression of Ptch1, Gas1, Cdon, and Boc in the middle ear joints (black brackets), annular ligaments (red brackets, B-F), and stapedial footplate (arrowheads, B-F). i, incus; $m$, malleus; s, stapes; $A$, anterior; L, lateral. 
regions of the vestibule (Fig. 5 G-G"), outer sulcus cells derived from the lesser epithelial ridge, and strial marginal cells derived from the cochlear epithelium (Fig. $5 \mathrm{H}-\mathrm{H}^{\prime}$ ). Boc expression was down-regulated in the sensory regions of the vestibule at PO (Fig. $5 \mathrm{I}-\mathrm{l}$ "), but was continuously expressed in the GER (prospective Kolliker's organ), Hensen and Claudius cells, and outer sulcus region (Fig. $5 \mathrm{~J}-\mathrm{J}$ '). Our results indicated that $\mathrm{Hh}$ co-receptor expression domains are generally maintained during inner ear development from E15.5 to P0. However, because expression of Shh or Ptch1 was not detected in the inner ear at P0, it is unclear whether Hh co-receptors contribute to inner ear development by mediating Shh signaling.

\section{Expression patterns of $\mathrm{Hh}$ co-receptors in the developing middle ear}

The mammalian middle ear consists of a chain of ossicles, including the malleus, incus, and stapes. These ossicles articulate in response to sound vibrations from the outer ear by malleoincudal and incudostapedial joints. The stapes is attached to the cochlea by annular ligaments (Thompson et al., 2012). Since Shh has been shown to be associated with middle ear development (Jeong et al., 2004), we examined the expression of $\mathrm{Hh}$ co-receptors in the developing middle ear at E15.5 (Fig. 6). Aggrecan (Acan) was expressed in the developing cartilaginous ossicles of the middle ear (Fig. $6 \mathrm{~A}, \mathrm{G}$ ), whereas growth differentiation factor (Gdf5) was expressed in the joints between ossicles and in the annular ligaments between the stapes and cochlea (Fig. 6B, H, brackets) (Hwang and $\mathrm{Wu}, 2008)$. The region of Ptch 1 expression was located between those of Acan and Gdf5 (Fig. 6 C,I). Expression domains of Gas1 and Cdon were similar to that of Gdf5 in the joints and annular ligaments, but were broader and encompassed both Gdf5 and Ptch 1 expression domains (Fig. 6 D,E,J,K). In contrast, Boc was weakly expressed in a pattern similar to that of Ptch1 around the joints and annular ligaments (Fig. $6 \mathrm{E}, \mathrm{L}$ ). Interestingly, none of the $\mathrm{Hh}$ co-receptors were expressed in the developing cartilaginous ossicles of the middle ear. It is currently unclear as to what extent Hh signaling contributes to the development of middle ear ossicles and joints. However, because Hh signaling has been shown to play a role in the formation of the temporomandibular joint of the jaw (Purcell et al., 2009), it is possible that Hh co-receptors mediate $\mathrm{Hh}$ function to pattern the joints and ligaments in the middle ear.

\section{Summary}

In this study, we analyzed spatial and temporal expression patterns of Hh co-receptors, including Gas1, Cdon, and Boc, in the developing inner and middle ear. Each co-receptor was expressed in specific domains in the inner and middle ear that were generally maintained throughout development, although some specific details changed upon cellular differentiation and morphogenetic change. Our results suggest that $\mathrm{Hh}$ co-receptors play essential roles in mediating the functions of Shh, and possibly other Hh ligands, which are required for ear development by forming distinct combinations of receptor complexes in a space- and time-specific manner.

\section{Materials and Methods}

\section{Animals}

We purchased ICR pregnant mice from KOATECH (South Korea) and harvested the embryos at E9.5, E10.5, E11.5, E15.5, and postnatal day (P)0.
All animals were handled in accordance with the Guidelines for the Care and Use of Laboratory Animals of Yonsei University College of Medicine.

\section{In situ hybridization}

Antisense RNA probes against Acan, Gdf5 (Hwang and Wu, 2008), Atoh1, Ptch1, Shh (Bok et al., 2013), Bmp4, Lfng (Morsli et al., 1998), Boc [(+2522-+3678), NM_172506.2], Cdon [(-127-+949), NM_021339.2], Foxg1 [(+2428-+3465), NM_001160112.1], and Gas1 [(+1399-+1878), NM_008086.2] were labeled with digoxigenin (Roche, Mannheim, Germany). In situ hybridization was performed as previously described (Morsli et al., 1998). The micrographs of gene expression patterns were taken using OLYMPUS BX40 and Leica DM2500 optical microscopes.

\section{Acknowledgements}

We thank Dr. Jong-Sun Kang for plasmids encoding Cdon and Boc. This work was supported by National Research Foundation of Korea grants 2017R1A2B3009133 and 2016R1A5A2008630 (to J.B.) and grant 2016R1A6A3A11932191 (to J.S.).

\section{References}

ALLEN, B.L., SONG, J.Y., IZZI, L., ALTHAUS, I.W., KANG, J.S., CHARRON, F. KRAUSS, R.S. and MCMAHON, A.P. (2011). Overlapping roles and collective requirement for the coreceptors $\mathrm{GAS} 1, \mathrm{CDO}$, and $\mathrm{BOC}$ in $\mathrm{SH}$ pathway function Dev Cell 20: 775-787.

BOK, J., BRONNER-FRASER, M. and WU, D.K. (2005). Role of the hindbrain in dorsoventral but not anteroposterior axial specification of the inner ear. Development 132: 2115-2124.

BOK, J., DOLSON, D.K., HILL, P., RUTHER, U., EPSTEIN, D.J. and WU, D.K. (2007) Opposing gradients of Gli repressor and activators mediate Shh signaling along the dorsoventral axis of the inner ear. Development 134: 1713-1722.

BOK, J., ZENCZAK, C., HWANG, C.H. and WU, D.K. (2013). Auditory ganglion source of Sonic hedgehog regulates timing of cell cycle exit and differentiation of mammalian cochlear hair cells. Proc Natl Acad Sci USA 110: 13869-13874.

BRISCOE, J. and THEROND, P.P. (2013). The mechanisms of Hedgehog signalling and its roles in development and disease. Nat Rev Mol Cell Biol 14: 416-429.

BROWN, A.S. and EPSTEIN, D.J. (2011). Otic ablation of smoothened reveals direct and indirect requirements for Hedgehog signaling in inner ear development. Development 138: 3967-3976.

CAMP, D., CURRIE, K., LABBE, A., VAN MEYEL, D.J. and CHARRON, F. (2010). Ihog and Boi are essential for Hedgehog signaling in Drosophila. Neural Dev 5: 28.

HWANG, C.H. and WU, D.K. (2008). Noggin heterozygous mice: an animal model for congenital conductive hearing loss in humans. Hum Mol Genet 17: 844-853.

IZZI, L., LEVESQUE, M., MORIN, S., LANIEL, D., WILKES, B.C., MILLE, F., KRAUSS R.S., MCMAHON, A.P., ALLEN, B.L. and CHARRON, F. (2011). Boc and Gas1 each form distinct Shh receptor complexes with Ptch1 and are required for Shhmediated cell proliferation. Dev Cell 20: 788-801.

JEONG, J., MAO, J., TENZEN, T., KOTTMANN, A.H. and MCMAHON, A.P. (2004) Hedgehog signaling in the neural crest cells regulates the patterning and growth of facial primordia. Genes Dev 18: 937-951.

MARTINELLI, D.C. and FAN, C.M. (2007). Gas1 extends the range of Hedgehog action by facilitating its signaling. Genes Dev 21: 1231-1243.

MORSLI, H., CHOO, D., RYAN, A., JOHNSON, R. and WU, D.K. (1998). Development of the mouse inner ear and origin of its sensory organs. J Neurosci 18: 3327-3335.

PURCELL, P., JOO, B.W., HU, J.K., TRAN, P.V., CALICCHIO, M.L., O'CONNELL, D.J., MAAS, R.L. and TABIN, C.J. (2009). Temporomandibular joint formation requires two distinct hedgehog-dependent steps. Proc Natl Acad Sci USA 106 18297-18302.

RICCOMAGNO, M.M., MARTINU, L., MULHEISEN, M., WU, D.K. and EPSTEIN, D.J. (2002). Specification of the mammalian cochlea is dependent on Sonic hedgehog. Genes Dev 16: 2365-2378

SON, E.J., MA, J.H., ANKAMREDDY, H., SHIN, J.O., CHOI, J.Y., WU, D.K. and BOK J. (2015). Conserved role of Sonic Hedgehog in tonotopic organization of the avian basilar papilla and mammalian cochlea. Proc Natl Acad Sci USA 112: 3746-3751.

SONG, M.H., CHOI, S.-Y., WU, L., OH, S.-K., LEE, H.K., LEE, D.J., SHIM, D.-B., 
CHOI, J.Y., KIM, U.-K. and BOK, J. (2011). Pou3f4 deficiency causes defects in otic fibrocytes and stria vascularis by different mechanisms. Biochem Biophys Res Commun 404: 528-533.

TATEYA, T., IMAYOSHI, I., TATEYA, I., HAMAGUCHI, K., TORII, H., ITO, J. and KAGEYAMA, R. (2013). Hedgehog signaling regulates prosensory cell properties during the basal-to-apical wave of hair cell differentiation in the mammalian cochlea. Development 140: 3848-3857.

TENZEN, T., ALLEN, B.L., COLE, F., KANG, J.S., KRAUSS, R.S. and MCMAHON,
A.P. (2006). The cell surface membrane proteins $\mathrm{Cdo}$ and Boc are components and targets of the Hedgehog signaling pathway and feedback network in mice. Dev Cell 10: 647-656.

THOMPSON, H., OHAZAMA, A., SHARPE, P.T. and TUCKER, A.S. (2012). The origin of the stapes and relationship to the otic capsule and oval window. Dev Dyn 241: 1396-1404.

WU, D.K. and KELLEY, M.W. (2012). Molecular mechanisms of inner ear development. Cold Spring Harb Perspect Biol 4: a008409. 


\section{Further Related Reading, published previously in the Int. J. Dev. Biol.}

Single cell analysis of the inner ear sensory organs

Ofer Yizhar-Barnea and Karen B. Avraham

Int. J. Dev. Biol. (2017) 61: 205-213

https://doi.org/10.1387/ijdb.160453ka

\section{Limited inner ear morphogenesis and neurosensory development are possible in the absence of GATA3}

Jeremy S. Duncan, Kim-Chew Lim, James D. Engel and Bernd Fritzsch

Int. J. Dev. Biol. (2011) 55: 297-303

https://doi.org/10.1387/ijdb.103178jd

\section{Patterning and cell fate in ear development}

Berta Alsina, Fernando Giraldez and Cristina Pujades

Int. J. Dev. Biol. (2009) 53: 1503-1513

https://doi.org/10.1387/ijdb.072422ba

Patterning and morphogenesis of the vertebrate inner ear

Jinwoong Bok, Weise Chang and Doris K. Wu

Int. J. Dev. Biol. (2007) 51: 521-533

https://doi.org/10.1387/ijdb.072381jb

Mouse models to study inner ear development and hereditary hearing loss Lilach M. Friedman, Amiel A. Dror and Karen B. Avraham

Int. J. Dev. Biol. (2007) 51: 609-631

https://doi.org/10.1387/ijdb.072365lf

Axial patterning in the developing vertebrate inner ear

Tanya T. Whitfield and Katherine L. Hammond

Int. J. Dev. Biol. (2007) 51: 507-520

https://doi.org/10.1387/ijdb.072380tw

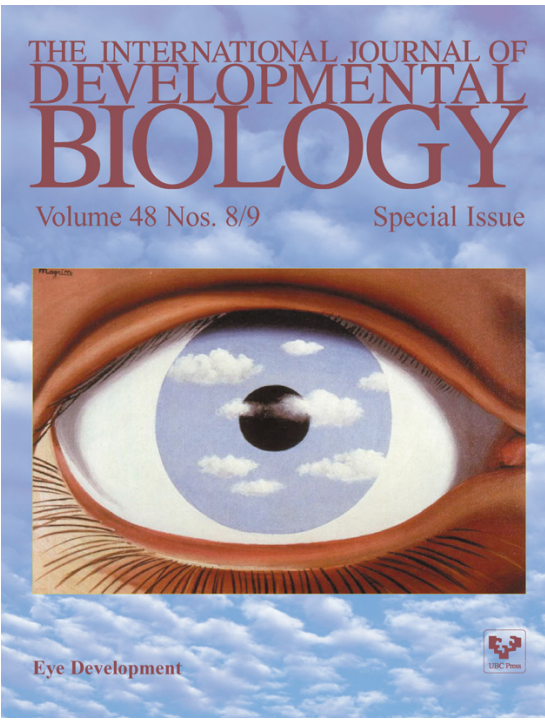

5 yr ISI Impact Factor $(2013)=2.879$
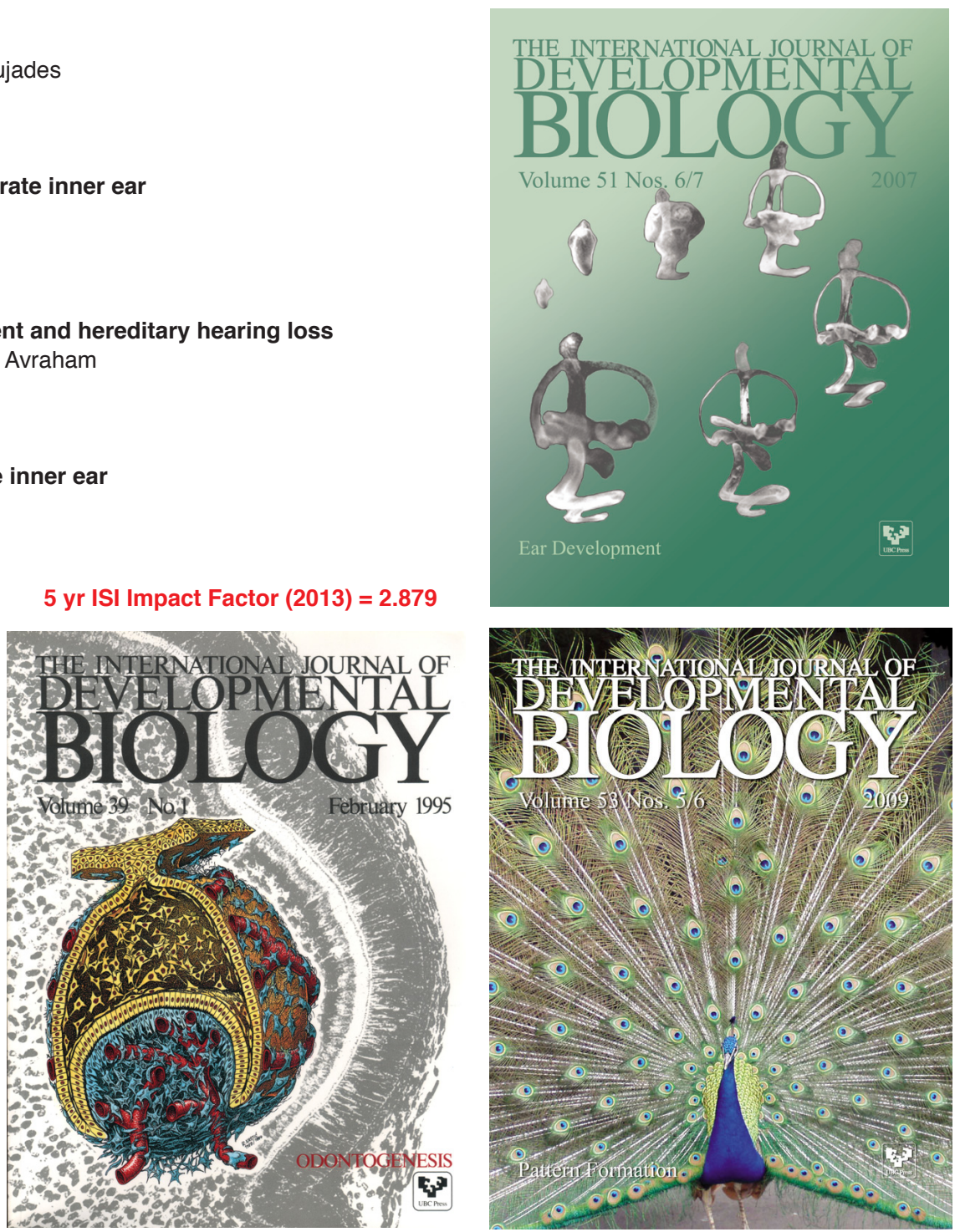